\title{
TUBERCULOSIS GENITAL FEMENINA
}

\author{
Doctores Bernardo Botero Peláez*, Jesús Montes Sáenz**, \\ Oscar Acebedo Ferrer ${ }^{* * *}$. Antonio J. Giraldo G. ****
}

Hace algunos años, el diagnóstico de la tuberculosis genital era difícil y muchas veces imposible para nosotros. Los hallazgos quirúrgicos - verdaderas sorpresas- se sucedían inexorablemente. Esta serie de fracasos diagnósticos nos llevó a pensar más en esta entidad, obligándonos a un mejor análisis de las pacientes, especialmente aquellas jóvenes, estériles, con dolor pélvico y trastornos del ciclo, que presentaban además cérvix sano, anexos palpables o masas anexiales sin antecedentes de infección séptica o gonocóccica.

Gracias al incremento dado al estudio de la esterilidad por la I.F.A. en los últimos $\mathrm{I} 3$ años, por medio de los consultorios especializados de esterilidad en donde se realizan rutinariamente biopsias y curetajes diagnósticos, se han venido a comprobar índices muy altos de tuberculosis geni- tal, especialmente de las formas subclínicas en las cuales sólo usualmente se llega al diagnóstico por estos medios.

Material. El material para el presente trabajo consta de 64 casos de tuberculosis genital, recopilación en el período I956-1963 primer semestre. De éstos casos, 53 pertenecen a la consulta de la Cátedra de Ginecología del Hospital Universitario de Caldas y ir casos a nuestra consulta privada.

De acuerdo con la localización que nos sirvió para hacer el diagnóstico, los dividimos así: tuberculosis endometrial, 29 casos $(45.3 \%)$; tuberculosis de las trompas, 33 casos $(51.6 \%)$. Al diagnóstico se llegó exclusivamentc por cultivo en dos casos $(3.1 \%)$ ya que en ellos no se encontraron lesiones histológicas de tuberculosis genital a pesar de biopsias repetidas.

\footnotetext{
* Jefe del Departamento de Obstetricia y Ginecología. Hosvital Universitario de Caldas.

क* Director de Unidad Ginecológica. H. U. C.

**** Coordinador del Departamento Obstetricia y Ginecología. H. U. C.

$* * *$ Auxiliar del Departamento de Obstetricia y Ginecología. H. U. C.
} 
El total de casos de endometritis tuberculosa fue de 42 , por haberse comprobado posteriormente localización endometrial en 13 casos de tuberculosis tubaria.

\section{CUADRO 1}

Motivos de consulta. El principal motivo de consulta lo constituyó el dolor pélvico, siguiendo en orden de frecuencia la esterilidad y los trastornos del ciclo; de éstos 7 (10.9\%), corresponden a pacientes en amenorrea.

CUADRO 3

\begin{tabular}{ll}
\hline & No \\
\hline Dolor pélvico & $4 \mathrm{I}$ \\
Esterilidad & 30 \\
Trastornos del ciclo & 25 \\
Otros motivos & 29 \\
\hline
\end{tabular}

Paridad. En 28 pacientes (43.7\%) había historia de I49 embarazos, de los cuales i26 fueron a término y 23 terminaron en aborto.

En el cuadro número 4 se muestra en detalle la historia obstétrica de estas pacientes.

CUADRO 4

\begin{tabular}{rrrr}
\hline I & I & I & \\
8 & 2 & I & I \\
3 & 3 & 3 & \\
I & 3 & 2 & I \\
I & 4 & & 4 \\
I & 4 & 4 & \\
2 & 6 & 6 & \\
3 & 7 & 7 & \\
I & 9 & 9 & \\
I & 9 & 8 & I \\
I & 9 & 6 & 3 \\
I & I2 & 9 & 3 \\
I & I3 & I2 & I \\
I & I5 & I3 & 2 \\
I & I9 & I2 & 7 \\
\hline
\end{tabular}


Esta notable frecuencia de embarazos en nuestro grupo contrasta con las cifras reportadas por Sutherland ${ }^{1}$ de un $12.9 \%$ de embarazos previos al diagnóstico de tuberculosis genital, correspondiendo el porcentaje obtenido por nosotros al $43.7 \%$. Basándose en su estadística y en la literatura acumulada a este respecto, este autor reafirma la baja fertilidad en mujeres que presentan localización genital de la tuberculosis.

Puede presumirse que un buen número de estos embarazos previos coexistieron con endometritis tuberculosa y trompas permeables, posibilidad que ha sido demostrada entre nosotros por Botero y Restrepo ${ }^{2}$.

Embarazo ectópico. Una condición poco frecuente es la coexistencia del embarazo ectópico con la tuberculosis tubaria, Darvin-Power, O'Dwyer y Leslie en I955, estudian un caso y revisan la literatura mundial encontrando 52 casos reportados y sólo 2 en la literatura británica ${ }^{3}$.

A. Varela Núñez, cita una estadística de Stevenson y Wharton en la cual se reporta un caso de tuberculosis genital, coexistiendo con embarazo ectópico, sobre un total de 402 casos de salpingitis tuberculosa y $5 \mathbf{1 0}$ casos de embarazo tubario ${ }^{4}$.

En 3 de nuestros casos $(4.7 \%)$, la tuberculosis de las trompas coexistió con embarazo ectópico. De ellas 2 eran estériles primarias y una tenía historia de un embarazo a término. El examen histológico comprobó en 2 casos la tuberculosis en la trompa donde se implantaba el huevo y en el caso restante, sólo en la trompa opuesta.

Se reconoce que la tuberculosis genital no es causa de esterilidad absoluta. Con el uso de los antibióticos y quimioterápicos cada vez se reportan más embarazos normales y ectópicos en pacientes que han recibido tratamiento ${ }^{5}$.

Un caso de embarazo ectópico, (aborto tubario), no incluído en el grupo anterior, corresponde a una paciente estéril primaria de 7 años cuyo diagnóstico de tuberculosis genital se confirmó por cultivo inmediatamente después de haber recibido tratratamiento para tuberculosis pulmonar durante un año. Las biopsias de endometrio tomadas al principio, durante y al final del segundo tratamiento fueron negativas y una histerosalpingografía mostró trompas normales, tres meses antes de presentar el aborto tubario. El estudio histológico de una biopsia de pabellón tubario no reveló en ese momento signos de tuberculosis.

Esterilidad. El total de pacientes estériles fue de $36(56.2 \%)$; de éstas, $27(42.2 \%)$ estériles primarias y 9 (14.1\%) estériles secundarias.

Del grupo anterior pertenecían a la consulta de esterilidad $30(46.8 \%)$.

CUADRO 5

\begin{tabular}{lrr}
\hline & No & \multicolumn{1}{c}{$\%$} \\
\hline Esterilidad primaria & 27 & 42.2 \\
Esterilidad secundaria & 9 & I4.I \\
Total de esterilidad & 36 & \\
\hline
\end{tabular}


Sobre un total de 3 ro pacientes de la conculta hospilataria de esterilidad, 2I $(6.7 \%)$ tuvieron diagnóstico de tuberculosis genital. Sobre un total de 330 pacientes de la consulta privada de esterilidad de uno de nosotros (B. B. P.), $6(1.8 \%)$ tuvieron diagnóstico de tuberculosis genital. Estas cifras ponen de manifiesto las diferencias socio-económicas de los grupos en mención.

CUADRO 6

No No \%

$\begin{array}{lllll}\text { Consulta hospitalaria } & 3 \mathrm{IO} & 2 \mathrm{I} & 6.7\end{array}$ $\begin{array}{llll}\text { Consulta privada } & 330 & 6 & \mathbf{1} .8\end{array}$

Trastorno del ciclo. Eran eumenorreicas 25 pacientes $(39 \%)$; en 39 (6r.0\%) acusaban trastornos del ciclo consistentes por orden de frecuencia en: oligomenorrea, amenorrea, polimenorrea, metrorragia, hipo e hipermenorrea. Cifras semejantes presenta Murray en su clásico trabajo sobre tuberculosis genital ${ }^{6}$.

CUADRO 7

\begin{tabular}{lrr}
\hline & Ne & $\%$ \\
\hline Eumenorrea & 25 & 39.0 \\
Oligomenorrea & 9 & 14.0 \\
Amenorrea & 7 & $\mathbf{1} .0$ \\
Polimenorrea & 6 & 9.4 \\
Metrorragia & 6 & 9.4 \\
Hipomenorrea & 4 & 6.3 \\
Hipermenorrea & 3 & 6.3 \\
Menopausia & 4 & 6.3 \\
$\quad$ Totales: & 64 & $\mathbf{1 0 0 . 0} \%$ \\
\hline
\end{tabular}

Menopausia. La tuberculosis genital es poco frecuente en la menopausia. Se dice que la curación espontánea de la endometritis tuberculosa explicaría la poca incidencia de la enfermedad en la menopausia, siendo precisamente la época en la cual se practican más biopsias y curetajes diagnósticos ${ }^{7}$. Por otra parte, el poco aflujo sanguíneo y la atrofia endometrial serían condiciones poco favorables para el desarrollo del proceso tuberculoso ${ }^{7}$.

Barbour ${ }^{7}$, reporta 9 casos de tuberculosis y menopausia en ro6 pacientes recopilados en un período de 30 años. Las edades oscilaban entre 4I y 53 años.

En nuestra casuística tenemos 4 menopáusicas $(6.2 \%)$; la primera de 43 años había tenido 9 hijos y entró en menopausia inmediatamente después del último parto, 9 años antes. Tenía localización endometrial y cervical con necrosis caseosa de todo el endometrio.

La segunda 49 años había tenido 7 hijos; inició la menopausia 29 años atrás, es decir a los 30 años de edad. Tenía endometrio proliferativo.

La tercera de 56 años estuvo casada 29 años y no tuvo hijos; entró en menopausia a los 35 años. Consultó por metrorragias escasas de 3 meses de duración. El endometrio era atrófico.

La cuarta de 56 años, había tenido I3 partos y 2 abortos. Consultó por metrorragias escasas y prolapso geni- 
tal. Inició la menopausia 8 años atrás. Tenía endometrio hiperplásico.

En contraste con la opinión de algunos $^{8}$ que reportan metrorragias graves en sus pacientes menopáusicas, sólo 2 de nuestras pacientes tuvieron metrorragias y éstas fueron leves.

Estado del aparato genital. El cérvix estaba sano en 42 casos $(65.6 \%)$ y en $16(25.0 \%)$ había patología cervical; no se consignó su estado en 6 mesas $(9.4 \%)$. Se presentó tuberculosis del cérvix en 3 casos $(4.7 \%)$ coexistiendo siempre con localización endometrial. Las lesiones eran hipertróficas en todos los casos; se hizo el diagnóstico de carcinoma cervical en 2 casos y en el restante, debido a las condiciones especiales de la paciente se presumió la tuberculosis genital con localización en cérvix.

El útero estaba en posición normal en 32 casos $(50.0 \%)$; en retroversión en $18(28.1 \%)$; en lateroposición en I4 $(2 \mathrm{I} .9 \%)$. Había prolapso genital en 3 casos $(4.7 \%)$. El útero no fue delimitable en $\mathrm{I} 2$ casos ( $18.7 \%$ ).

Los anexos eran palpables en i I ca$\operatorname{sos}(17.2 \%)$; había masas anexiales en $28(43.7 \%)$; anexos no palpables en $22(34.4 \%)$; no fue consignado el estado de los anexos en 3 casos.

Diagnóstico. En nuestro grupo, 27 pacientes $(42.2 \%)$ tenían diagnóstico presuntivo de tuberculosis genital. En 33 pacientes que fueron operadas se hizo diagnóstico macroscópico correcto en 22.
Cabe anotar que en un buen número de pacientes las lesiones del aparato genital eran más o menos ostensibles, lo cual facilitó el diagnóstico.

\section{Anatomía patológica. Se estudiaron} 42 biopsias de endometrio cuyo resultado se consigna en el cuadro número 8. El predominio del endometrio secretor, se debe al hecho de que rutinariamente tomamos las biopsias en el premenstruo.

CUADRO 8

\begin{tabular}{lr}
\hline & x: \\
\hline Secretor & I8 \\
Proliferativo & $\mathrm{I} 6$ \\
Hiperplásico & 6 \\
Atrófico & $\mathrm{I}$ \\
Destrucción completa & $\mathrm{I}$ \\
\hline
\end{tabular}

Eritrosedimentación. Era normal en $25(39.0 \%)$; acelerada en 28 $(43.7 \%)$; no se consignó en II casos $(17.2 \%)$. Esta prueba complementaria de diagnóstico tiene un valor relativo?.

Histerosalpingografía. Este medio diagnóstico ha sido usado con cautela entre nosotros cuando presumimos el diagnóstico de tuberculosis genital, a pesar de reconocer su importancia, por el peligro de reactivar procesos anexiales como nos ha ocurrito en ocasiones.

Persuflación. Se practicó persuflación tubaria con dióxido de carbono 
en 3 pacientes previo el diagnóstico de tuberculosis genital; en 2, la gráfica fue de estenosis y en una de obstrucción completa.

En una paciente que había tenido un aborto tubario después de un año de tratamiento para tuberculosis genital, se practicó persuflación un mes después de la intervención habiéndose obtenido una gráfica de permeabilidad pero a una presión superior a lo normal.

Cultivos. Sólo recientemente hemos incorporado al estudio de la tuberculosis genital como métodos de rutina el cultivo y la inoculación de sangre menstrual, macerado endometrial y secreciones uterinas. Citamos aquí dos cultivos positivos en pacientes a las cuales no se les pudo comprobar localización endometrial, a pesar de haberles practicado biopsias repetidas.

Tratamiento. Recibieron tratamiento completo 35 pacientes $(54.0 \%)$; fue necesario operar $3(4.6 \%)$ porque al final del tratamiento médico, conservazan masas anexiales dolorosas. Figuran con tratamiento incompleto $29(45.3 \%)$ bien sea porque no volvieron a consulta o porque aún continúan en él.

Anotamos lo que siempre se ha comprobado en el tratamiento de la tuberculosis genital y es la repercusión inmediata y favorable sobre los trastornos del ciclo.

Sumario y conclusiones. Se estudian 64 casos de tuberculosis genital femenina. Se encuentra localización tubaria en 33 casos y endometrial en 29. Se hace el diagnóstico por cultivo en 2 casos.

En 52 casos la edad está comprendida entre los 20 y los 39 años correspondiendo al período genital activo.

Se encuentra gran fertilidad en 28 pacientes, con un total de I49 embarazos y 126 partos.

El principal motivo de Consulta es el dolor pélvico, siguiendo en orden de frecuencia la esterilidad y los trastornos del ciclo.

Se presentan 4 casos en la menopausia coexistiendo con tuberculosis genital, localización endometrial.

Se presentan 3 casos de embarazo ectópico coexistiendo con tuberculosis de las trompas. Se presenta otro caso de embarazo ectópico después del tratamiento de la tuberculosis genital.

El $39.0 \%$ de las pacientes son eumenorreicas; el $54.0 \%$ acusan trastornos del ciclo.

El alto índice de tuberculización del Departamento de Caldas está en relación con la prevalencia de tuberculosis genital femenina que se consigna en este trabajo.

Debe presumirse, tuberculosis genital en mujeres jóvenes que presentan dolor pélvico, esterilidad, alguna patología pélvica y cérvix sano. 


\section{BIBLIOGRAFIA}

1. SUTHERLAND A.: Genital tuberculosis in women. Am. J. Obst. and Gyn., 79 (3): 488. March, 1960.

2. BOTERO J. y RESTREPO J.: Tuberculosis endometrial y embarazo. Antioquia médica II (5): 304-311. Junio, 1961.

3. GORDON MILLAR W. and col.: Tubal pregnancy after treatment of genital tuberculosis. The J. Obst. and Gyn. of Brit. Emp. 65 (5): October, 1958.

4. VARELA NUÑEZ A.: Tubal pregnancy folloming treated genital tuberculosis. Am. J. Obst. and Gyn 82 (5): 1163. Nov., 1961.

5. HALBRECHT I.: Heald genital tuberculosis. A new etiologig factor in ectopic pregnancy. Gyn. and Obst. Am. J. 10 (1). 73. Jul., 1957.

7. BARBOUR E. M.: Post-menopausal endometrial tuberculosis. The J. of Obst. and Gyn. of the Brit. Emp. 67 (6): 1009. Dec. 1960.

8. COUNTRY L. et OUTRES: Métrorragies graves de la ménopause par tuberculose utérine. Bulletin de la Federation des Sociétés de Gynecologie et D'obstetrique 15 (1): 57-58. Jan, Mars. 1963.

9. DI PAOLA G.: Endometritis tuberculosa y alteraciones menstruales. Obst. y Gin. Lat. Am. 8 (9): 436. Sept., 1960.

Jan, Mars., 1963. 